\title{
Imaging the Life and Death of mRNAs in Single Cells
}

\author{
Jeffrey A. Chao ${ }^{1}$ and Timothée Lionnet ${ }^{2}$ \\ ${ }^{1}$ Friedrich Miescher Institute for Biomedical Research, $\mathrm{CH}-4058$ Basel, Switzerland \\ ${ }^{2}$ Institute for Systems Genetics, Department of Cell Biology, NYU Langone Health, New York, New York 10016 \\ Correspondence: jeffrey.chao@fmi.ch; timothee.lionnet@nyumc.org
}

\section{SUMMARY}

RNA plays a central role in gene expression from its transcription in the nucleus through translation and degradation in the cytoplasm. Technological advances in fluorescent microscopy and labeling methodologies have made it possible to detect single molecules of RNA in both fixed and living cells. Here, we focus on the recent developments in RNA imaging that have allowed quantitatively measuring the lives of individual transcripts from birth to death and all the events in between in single cells and tissues. Direct observation of RNAs within their native cellular environment has revealed a complex layer of spatial and temporal regulation that has profoundly impacted our understanding of RNA biology.

\section{Outline}

1 Visualizing RNA at the single-cell and single-molecule scale

2 smFISH reveals the basic features of gene expression regulation

3 Localization as a gene expression regulator

4 Increasing spatial resolution: Toward structural resolution of mRNPs with light microscopy

5 Optimized in situ techniques to visualize and quantify the diversity of RNAs and their processing at the singlemolecule level

6 Increasing RNA visualization throughput
7 Imaging RNA beyond cultured cells: Recent developments for single-molecule RNA imaging in tissue

8 Imaging RNAs in living cells

9 Transcription

10 Splicing

11 Localization

12 Translation

13 mRNA decay

14 Outlook

References 


\section{VISUALIZING RNA AT THE SINGLE-CELL AND SINGLE-MOLECULE SCALE}

The ability to visualize messenger RNA (mRNA) provides a powerful means to probe its role in gene expression, and methods to directly image mRNAs in situ have long been sought (Harrison et al. 1973). The sensitivity and resolution of these techniques are continuously improving. Initial radioactivity-based methods have largely been replaced by fluorescence microscopy, and it is now possible to accurately measure the number and position of individual molecules of RNA in cells and tissue. Detection of RNAs in situ allows these molecules to be studied in their native context and bypasses common biochemical steps of extraction and purification. These procedures have variable efficiency and are prone to artifacts because RNAs are exquisitely sensitive to RNases (endogenous or environmental). Different RNA species vary in their sensitivity to degradation, which can engender serious biases in data collection and interpretation. Imaging is also applicable to limited samples (e.g., rare cell types) that are challenging to characterize using biochemical approaches that rely on ensemble measurements. The main advantage of mRNA imaging, however, resides in its inherent ability to capture RNAs within the environment of the cell or tissue, thereby allowing RNAs to be readily assigned to their relevant organelles or subcellular position. This information is extremely valuable because RNA location usually provides immediate clues to RNA function. The importance of obtaining gene expression data at the single-cell resolution is becoming more apparent, and imaging naturally provides cellular resolution, making it possible to identify specific cell types and capture cell-to-cell variability. Finally, genetically encoded fluorescent labels now enable live tracking of mRNA molecules, and visualizing sequences of events in living cells has provided fundamental insights into the life cycle of RNA. Here, we review the recent developments in single-molecule RNA imaging in fixed and living samples and outline the lessons learned on the way about the complex lives of RNAs.

\section{2 smFISH REVEALS THE BASIC FEATURES OF GENE EXPRESSION REGULATION}

Fluorescent in situ hybridization (FISH) relies on labeling a sample with fluorescent nucleic acid probes complementary to an RNA species of interest. After washing, only the probes bound to their RNA target remain, and the distribution of the fluorescent signal reflects that of the underlying RNAs. Although initial incarnations of the techniques used complementary DNA (cDNA) as a probe, progress in microscopy technology coupled with the use of DNA oligonucleotides coupled to fluorophores as probes has enabled tremendous increases in sensitivity. The first demonstration of single-molecule sensitivity FISH (smFISH) was published 20 years ago (Femino et al. 1998), using 50-nt-long DNA antisense probes (five to 10 different probes) containing four to five fluorophores. Although this technique worked robustly to detect single RNAs, a major barrier to its wide adoption was the requirement for each probe to be custom synthesized containing aminoallyl thymidine nucleotides and labeled with fluorescent dyes. The subsequent introduction of 20-nt probes coupled to a single fluorophore (25 to 40 different probes) that could readily be purchased from commercial sources (Raj et al. 2008) turned the technology into a popular tool that is now routinely used in a variety of laboratories, working on diverse sample types (Fig. 1A). Various image analysis tools can automatically detect the fluorescent spots emitted by individual RNA molecules and quantify their position and fluorescence intensity (Raj et al. 2008; Mueller et al. 2013; Trcek et al. 2017). The data typically collected in these experiments provides the absolute numbers of RNA molecules in each cell imaged, making the technique the gold standard in the field of quantitative gene expression. Sensitivity routinely ranges in the $80 \%-$ $100 \%$ range, with low levels of false positives (Raj et al. 2008).

Early work took advantage of the high-sensitivity measurements of single-cell expression levels offered by smFISH to test quantitative models of gene expression (Raj et al. 2006; Zenklusen et al. 2008; Taniguchi et al. 2010). The results obtained in a variety of model systems showed that the transcription process, which was long thought to be finely regulated and predictable, is actually highly stochastic (Lionnet and Singer 2012): transcripts are generated in bursts of activity interspersed by periods of inactivity. Although the average bursting probabilities are biologically regulated and differ between genes, individual transcripts in a cell are randomly expressed over time, which means that genetically identical cells can express dramatically different levels of the same mRNA (Fig. 1B). A unified understanding of the molecular regulation of bursting kinetics is still lacking, although different factors have been suggested to play a role, from chromatin accessibility at the promoter (Bai et al. 2010; Lionnet and Singer 2012) to transcriptioninduced supercoiling accumulation (Chong et al. 2014), enhancer looping (Bartman et al. 2016), and binding of transcription factors or the transcription machinery at transcription hubs (Cho et al. 2016).

Early attempts at multiplex smFISH experiments labeled distinct mRNA species in the same sample with unique fluorophore labels (Fig. 1A). These experiments provided a way to explore the correlation between the mRNA numbers from different genes across a cell population. Interestingly, little correlation was observed between the transcription levels of different genes, even between functionally related ones and alleles of the same gene (Raj et al. 2006; 
A

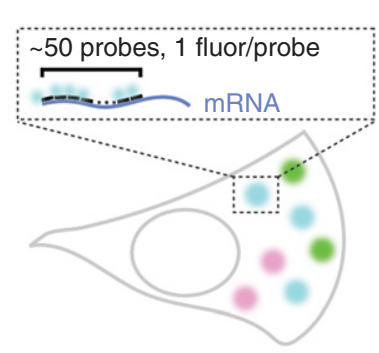

D Mutation detection

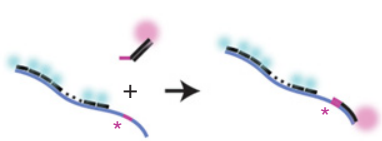

B

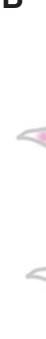

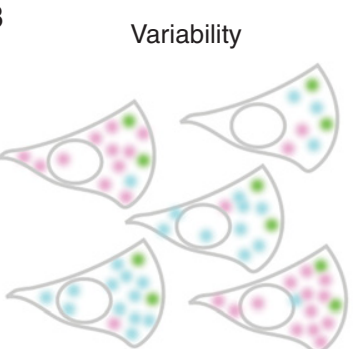

C

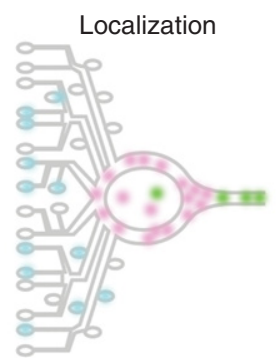

E Multiplexing with sequential hybridizations

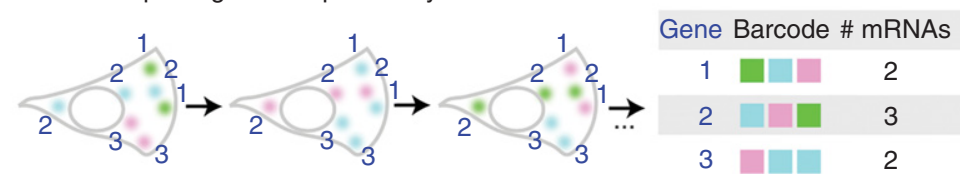

Figure 1. Single-molecule RNA fluorescent in situ hybridization (smFISH) and variants. (A) Principle of the technique: A probe library consisting of typically 50 20-mer oligonucleotides is hybridized to a sample. After washing excess probes, individual mRNAs appear as bright fluorescent spots. Multiplexing libraries in separate colors provides access to the expression of three separate genes in this example. $(B)$ smFISH provides access to naturally occurring variability within a cell population, also called noise. Here, the products of two genes (pink and blue) display high variability and are anticorrelated. $(C)$ smFISH provides access to the subcellular localization of mRNAs, separating for instance dendrite-targeted (blue) and axon-targeted mRNAs (green) from other species localized in this neuron's soma. $(D)$ Combining a "guide" probe library (blue spots) with a specifically designed toehold probe labeled in a separate color (pink spot) provides a reading of the fraction of a given species carrying a mutation of interest. $(E)$ Multiplexing via sequential hybridizations: RNAs in the same sample are hybridized sequentially to distinct library probes and imaged after each hybridization cycle. A given mRNA appears in a separate color at each cycle, and the sequence of colors detected at each mRNA position gives the molecule identity away.

Gandhi et al. 2011). The only genes displaying high correlation in their transcript levels were genes closely located on the genome, suggesting the importance of the chromatin environment in regulating expression. The immediate corollary of bursting is that expression levels between cells can vary substantially, setting obvious challenges to orderly cellular function. One simple strategy that seems to have evolved to generate precise mRNA levels from highly variable transcription sources is the temporal (or spatial) averaging of transcripts: If bursting occurs on a fast timescale (e.g., minutes) compared with the lifetime of its mRNA product (e.g., hours), the fluctuation of mRNA copy numbers are greatly attenuated compared with the variability of the transcription dynamics (Little et al. 2013). mRNA decay was recently shown to occur homogeneously in the cytoplasm, confirming its possible role as a dampener of expression noise (Horvathova et al. 2017). Besides its intrinsic importance as a fundamental constraint on cellular function, the control of expression variability in a cell population also has crucial consequences for disease. For instance, drug resistance in cancer cell lines has been shown to occur via transient expression of resistance genes (Shaffer et al. 2017). These transient bursts of expression eventually turn into stable expression profiles during drug treatment, indicating their capacity to function as a selection pressure.

\section{LOCALIZATION AS A GENE EXPRESSION REGULATOR}

Imaging provides immediate access to the spatial distribution of mRNAs in tissues and cell. Many studies have shown that the transcription, transport, and degradation of mRNA are used to generate spatial patterns of gene expression (Martin and Ephrussi 2009). The first step for most mRNAs involves export through the nuclear pore toward the cytoplasm where they can be translated. The regulation of nuclear export has been studied by various laboratories using smFISH. In principle, export from the nucleus could either amplify or buffer transcription-induced fluctuations of RNA levels depending on the relative kinetics of transcriptional bursting, export, and mRNA degradation. Indeed, a variety of scenarios have been observed that depend on the specific transcript (Bahar Halpern et al. 2015; Stoeger et al. 2016). As not all RNAs are destined to be translated, the localization of noncoding RNAs emerged as an important clue in their function. FISH experiments probing the localization of more 
than 60 long noncoding RNAs (lncRNAs) showed a wide variability of spatial patterns, from accumulation in nuclear foci to cytoplasmic localization, suggesting a wide range of function for these molecules (Cabili et al. 2015).

Developmental patterning provides striking examples of spatial restriction of mRNA expression at the tissue level, even before the advent of cellularization. In Drosophila embryos, $70 \%$ of mRNA species observed by FISH were found to have a regulated spatial pattern of expression (Lécuyer et al. 2007). Subcellular localization of mRNA transcripts is not restricted to embryogenesis, and perhaps the most striking example of spatial regulation is found in neurons. Because of their long processes, these cells face unique challenges in transporting gene expression products to their precise intended location. Part of this challenge is overcome by transporting select RNAs along dendrites and axons in which they can become translated near synapses on reception of the appropriate signal (Fig. 1C). Although most RNA species are found at or in the vicinity of the cell body, multiple examples of RNA localization into neuronal processes have been observed by FISH, from cultured neurons (Batish et al. 2012; Buxbaum et al. 2014) to intact brain tissue (Long et al. 2017). Together, these studies support a model of selective packaging of mRNA species into translationally repressive RNA-protein granules for their transport, and release from these structures then activates translation locally on demand. Interestingly, some of those structures can render RNAs invisible in standard smFISH experiments, presumably by blocking access of the probes to their target sequences (Buxbaum et al. 2014). A mild protease treatment can uncover those packaged transcripts and allow the study of the granule dynamics induced by neurostimulation. The recent discovery that one of the main activity-regulated neuronal genes, $A r c$, encodes a viral capsid-like protein that catalyzes its transfer to neighboring cells adds a new dimension to the role and regulation of RNA packaging and transport in neuronal function and communication (Ashley et al. 2018; Pastuzyn et al. 2018).

\section{INCREASING SPATIAL RESOLUTION: TOWARD STRUCTURAL RESOLUTION OF mRNPs WITH LIGHT MICROSCOPY}

As fluorescence microscopy technologies are improving at a fast pace, individual probes and molecules can now be localized with resolutions approaching the size of a macromolecular complexes $(\sim 10 \mathrm{~nm})$ (Sydor et al. 2015). Such progress opens the way to address structural questions left until now to the realm of electron microscopy. For instance, FISH combined with the stochastic optical reconstruction microscopy (STORM) super-resolution technique enabled the localization of individual probes in different colors tiling the same molecule of mRNA. The result was a spatial order of fluorescent spots consistent with the position of the probes along the sequence of a transcript (Lubeck and Cai 2012). A similar approach was recently taken to revisit the long-standing model positing that translating mRNAs adopt a circular conformation in which $5^{\prime}$ and $3^{\prime}$ ends of the transcripts are in close proximity, thereby enabling rapid reinitiation of translation (Wells et al. 1998). Surprisingly, those recent experiments challenge the notion of a circular conformation and suggest instead a somewhat extended mRNA (Adivarahan et al. 2017). In addition to these findings, recent work characterizing the RNA granules that form in the Drosophila oocyte using smFISH combined with structured illumination microscopy (SIM) showed a nonrandom spatial organization of the granules that contain the RNAs involved in instructing the fate of the future germ cells (Trcek et al. 2015). mRNAs of the same species tend to cluster together within a granule, and different RNAs occupy separate domains, pointing to a possible regulation of the granule organization. As the prevalence and importance of membrane-free granules and droplets is becoming more appreciated (Shin and Brangwynne 2017) and RNA binding proteins are disproportionately enriched in low complexity domains, smFISH will provide an important tool to probe the structure and assembly mechanism of these structures.

\section{OPTIMIZED IN SITU TECHNIQUES TO VISUALIZE AND QUANTIFY THE DIVERSITY OF RNAS AND THEIR PROCESSING AT THE SINGLE- MOLECULE LEVEL}

The smFISH technique relies on the binding of dozens (usually 20 to 100) of short oligonucleotide probes to the same transcript molecule. The large number of probes generate a signal amplification at the RNA location that allows detection of single RNA molecules from the background of single probes binding nonspecifically to other sites. Using multiple probes constitutes a limitation in many cases, for instance when imaging shorter RNA species, specific splicing isoforms or when single-nucleotide variants need to be resolved. Several adaptations to the smFISH technology now allow the detection of important classes of RNAs and variants. microRNAs (miRNAs) present a difficult challenge because of their short length, making it impossible to bind many probes. An optimized fixation protocol combined with specific probes made it possible to detect miRNAs in paraffin-embedded tissue, a notoriously difficult sample for RNA studies (Lu and Tsourkas 2009; Renwick et al. 2013). IncRNAs can be shorter than typical mRNAs and studies of localizations included sets of orthogonal probe library controls to validate the lower signals observed (Cabili et al. 2015). In the case of splicing iso- 
forms, the design of probes targeting different exons in distinct colors allows the detection of splicing events based on the observations of the colocalization of the two colors (Vargas et al. 2011; Waks et al. 2011). Finally, perhaps the most challenging questions to be addressed with smFISH are the detection of single-nucleotide variants (SNVs) and RNA editing events, such as adenosine to inosine conversion (Nishikura 2010). Detection of such minimal changes in RNA sequence have been made possible using a twocolor approach, in which probes in a first color label the conserved sequence while a unique toehold probe selective for the desired mutation is labeled with a second color (Fig. 1D) (Levesque et al. 2013; Mellis et al. 2017). Fluorescence in situ sequencing (FISSEQ, see below) is, in principle, capable of detecting individual mutations, but is technically challenging and typically only captures a small fraction $(<1 \%)$ of the transcripts in a sample (Lee et al. 2014).

\section{INCREASING RNA VISUALIZATION THROUGHPUT}

One of the main limitations of classic smFISH-based approaches is its throughput. Traditionally, different species of RNAs can be distinguished by labeling them with spectrally distinguishable fluorophores. However, the small number of separable fluorescent labels (typically about five channels across the visible spectrum) severely constrains the number of RNA species that can be probed simultaneously in a given sample, setting a hard "multiplexing barrier" to studying large numbers of RNAs. Methods to overcome this barrier are now expanding at an accelerated pace. The first attempt at spectral multiplexing used a barcode approach. Instead of labeling each RNA species in a single color, it was assigned a combination of colors. In the case of five possible labels, in which the traditional labeling approach would provide access to five species only, using combinations of two colors and three colors per RNA gives access to 20 species. This was first shown on nascent transcripts because sites of active transcription give bright signals caused by the accumulation of nascent transcripts in one location (Levsky et al. 2002), and further refined for individual transcripts (Lubeck and Cai 2012; Levesque and Raj 2013). These findings offered insights into nuclear organization and showed that the transcription state of genes within a chromosomal translocation were different from those in the normal copy of the chromosome. Interestingly, correlations in expression between genes on the same chromosome changed sign in the translocation relative to the normal genomic context (Levesque et al. 2013). A conceptually similar barcoding approach is based on sequential hybridizations. mRNAs are first hybridized to a first probe library and imaged. The probes are then washed away on the microscope and the sample is hybridized to a second library with distinct color assignments and imaged again. Multiple cycles are performed, yielding a series of images of the same sample. A given mRNA appears in each image with a different color according to the design of the probe library barcodes. The sequence of colors at each spot in the series of images allow the assignment of specific transcript identities (Fig. 1E) (Lubeck et al. 2014; Chen et al. 2015; Shah et al. 2016b). Importantly, the number of molecular species accessible in this sequential approach increases exponentially with the number of hybridization cycles; hundreds to thousands of mRNA species have been probed simultaneously with these techniques. These experiments have helped probe expression correlations at a deeper level, for instance, finding that some functionally related genes displayed positively correlated fluctuations, and that some groups (e.g., cell motility- vs. extracellular matrix-related genes) tended to be anticorrelated with one another in cultured cells.

One of the practical limits to throughput lies in the requirement for single-molecule resolution. If each molecule needs to be spatially separable from its neighbor to be reliably detected, this sets a limitation on experimentally tractable spot densities. Optical methods (super-resolution, expansion microscopy [Chen et al. 2016]) might help overcome this limitation. Novel image analysis tools able to quantify expression even for dense samples also provide increased throughput (Coskun and Cai 2016). Other avenues to multiplexing adapt sequencing-based methods are using in situ rolling circle amplification and sequencing of the transcripts in a cell; FISSEQ provides, in principle, access to genome-wide coverage (Lee et al. 2014). However, the practical limitations on achievable densities of amplicons limit capture to a small fraction of the transcriptome $(<1 \%)$. Another application of spatially resolved sequencing is spatial transcriptomics, in which a thin slice of sample is hybridized to a coverslip coupled to capture oligonucleotides. Each oligonucleotide carries a poly-T stretch to capture polyadenylated transcripts, as well as a sequence barcode that encodes its precise position along the coverslip (Ståhl et al. 2016). The resulting RNAs are reverse-transcribed and sequenced. This technique has genome-wide coverage and can deconvolve the initial position of each RNA molecule through the capture barcode of the initial capture strand. It remains limited in spatial resolution to $\sim 100 \mu \mathrm{m}$ pixel size and therefore provides a local average of RNA levels over a few cells. Although no technique is yet capable of providing genome-wide coverage at cellular and subcellular resolution with high efficiency, many of the technologies developed in recent years approach this goal, and combinations of image-based approach with sequencing provide exciting directions to investigate the spatiotemporal regulation of gene expression (Satija et al. 2015). 


\section{IMAGING RNA BEYOND CULTURED CELLS: RECENT DEVELOPMENTS FOR SINGLE- MOLECULE RNA IMAGING IN TISSUE}

Acquiring images deep within biological samples is challenging, as absorption and scattering by biological tissue can hinder the precise excitation and collection of relevant signals. This limitation is especially acute in the case of smFISH, in which weak signals from individual molecules need to be accurately detected. As imaging RNAs in their tissue of origin is a highly desirable application, adaptations of the classic FISH techniques have emerged. One way to overcome optical challenges in fixed tissue is to clear the tissue by chemically dissolving the light scattering compounds from the tissue, while retaining its spatial integrity. These techniques include the use of denaturants (urea), solvents (xylene), or surfactants, alone or in combination with hydrogels, that help retain the tissue architecture and can be expanded to increase the effective spatial resolution (Richardson and Lichtman 2015; Chen et al. 2016). Adaptations of the FISH protocol to these samples have achieved quantification of RNAs at depths of hundreds of microns in various sample types (Shah et al. 2016a; Long et al. 2017). The coupling with light sheet microscopy provides effective z-sectioning without excessive photobleaching typically encountered when using confocal microscopy. Finally, biological tissues typically generate high amounts of autofluorescence, even when cleared. This interferes with the detection of the typically weak signals emitted by individual RNA molecules hybridized to fluorescent oligonucleotide probes. New signal amplification strategies have emerged to increase the fluorescence emitted by individual mRNA molecules. These rely on the hybridization of branched DNA probes (Wang et al. 2012) or the hybridization chain reaction (HCR) in which a set of primary probes hybridize to their target RNA (Choi et al. 2014; Shah et al. 2016a,b). These probes carry a single-stranded DNA (ssDNA) tail that triggers the hybridization of multiple fluorescent probes, thereby amplifying the signal over that of a single probe. These techniques provide increased sensitivity in large tissue and have been combined with multiplexing to offer high barcode accuracy (Shah et al. 2016b).

\section{IMAGING RNAs IN LIVING CELLS}

Biological processes can be misinterpreted or undetected when one is unable to directly follow events unfolding in real time. A variety of fluorescent labeling methodologies have been devised that enable mRNAs to be detected and quantified in living cells (Czaplinski 2017). The most widely used of these techniques that enables detection of single molecules is based on the highly specific interaction between the MS2 bacteriophage coat protein (MCP) and its cognate RNA stem loop (Bertrand et al. 1998). In this approach, multiple copies of the MS2 stem loop are inserted into a transcript (usually the $3^{\prime}$ untranslated region [UTR]) of interest that can then be bound by MCP fused to a fluorescent protein. The accumulation of many fluorescent proteins on an individual transcript provides sufficient signal to allow robust detection of single RNA molecules. Using this system, many aspects of the mRNA life cycle have been investigated. However, the reliance on a single labeling technique inherently limits the complexity of the questions that can be addressed (Tutucci et al. 2018).

Recently, a number of orthogonal labeling strategies based on the PP7 bacteriophage coat protein (Larson et al. 2011), $\lambda \mathrm{N}$ bacteriophage (Daigle and Ellenberg 2007), U1A (Takizawa and Vale 2000), and BglG (Chen et al. 2009) have also been developed that can be used in combination for multicolor single-molecule RNA imaging when paired with spectrally distinct fluorescent proteins. The possibilities of multicolor RNA imaging experiments have just begun to be explored and have opened numerous new avenues of research. The following sections focus on the insights into the lives of RNAs that have been gained from two-color RNA imaging in living cells.

\section{TRANSCRIPTION}

Although fixed cell techniques such as smFISH make it possible to characterize cell-to-cell variability in mRNA and protein levels in genetically identical cells, multicolor live imaging provides a powerful tool to address the origin of expression fluctuations. The noise in gene expression can be separated into intrinsic (intracellular) and extrinsic (intercellular) factors that both contribute to the observed variability. Hocine and colleagues directly measured the contribution of intrinsic noise to transcription by labeling the two alleles of the essential gene MDN1, with either MS2 or PP7 stem loops in diploid yeast (Fig. 2A) (Hocine et al. 2013). By measuring the fluctuations in the number of MS2 and PP7-labeled transcripts over time in the same cell, the investigators found that the changes between alleles were not strongly correlated despite being transcribed in the same nucleus. These results highlight the stochastic nature of transcription and the contribution of intrinsic noise to this variability.

A similar approach was taken to measure the transcriptional activity of the pluripotency transcription factor Nanog, whose variability in expression regulates the balance between self-renewal and differentiation in mouse embryonic stem cells (Ochiai et al. 2014). Using transcription activator-like effector nuclease (TALEN)-mediated targeted integration, one Nanog allele was labeled by MS2 stem 
loops in its $3^{\prime}$ UTR and the other allele with PP7. Importantly, only a slight destabilization of the half-life of Nanog transcripts was observed on introduction of the stem loops, confirming that the introduction of these cassettes to endogenous genes can be minimally perturbing (Lionnet et al. 2011). Quantification of transcript numbers from the Nanog-MS2 and Nanog-PP7 alleles showed that $\sim 45 \%$ of the variability can be attributed to intrinsic noise, which again shows the highly variable nature of transcription.

Elucidating the molecular underpinning of bursting and its role in transcription variability has also been aided by two-color RNA imaging. Using Drosophila embryos as a model system, Fukaya and colleagues measured the transcription of two distinct MS2 and PP7-labeled genes controlled by the same snail shadow enhancer (Fukaya et al. 2016). When placed in a symmetric orientation with the enhancer equidistant from MS2 and PP7 reporter genes, these investigators observed similar bursting frequencies for both reporters. In the asymmetric orientation with the enhancer placed much closer to the PP7 reporter, the enhancer-proximal PP7 gene underwent sustained transcrip-

A
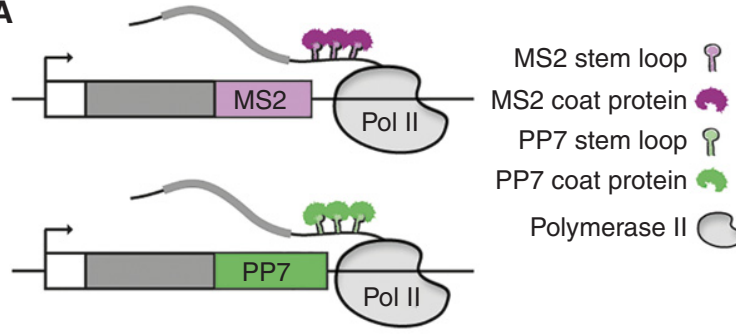

Polymerase $I 1$

B

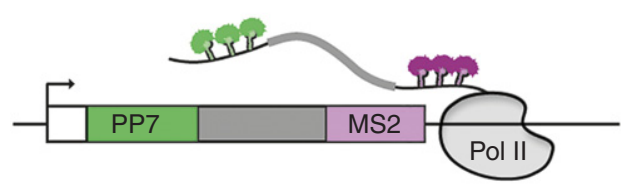

C

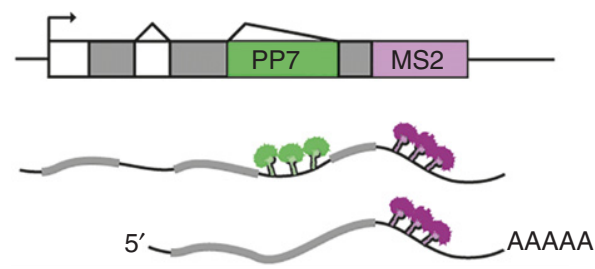

Figure 2. Single-molecule imaging of transcription and splicing in the nucleus of live cells. (A) Schematic representation of two-color labeling of alleles of the same gene with either MS2 or PP7 stem loops. $(B)$ Schematic representation of two-color intramolecular labeling of a transcript at the $5^{\prime}$ and $3^{\prime}$ ends to directly measure RNA Pol II transcription rates. $(C)$ Schematic representation of two-color intron and exon labeling for measurement of splicing. tion, while transcriptional bursts of the enhancer-distal MS2 reporter occurred less often. These results showed that competition between genes for the same enhancer can modulate bursting frequencies. The investigators then used their two-color RNA imaging system to characterize the coordination of transcription by a shared enhancer. If an enhancer is only able to interact with a single promoter at any given moment, then one would anticipate that MS2 and PP7 reporter transcription should be anticorrelated in time. Interestingly, the investigators observed that approximately two-thirds of bursts were coordinated between MS2 and PP7 and this synchrony was detected with enhancers of varying strength. Furthermore, addition of the gypsy insulator resulted as expected in fewer bursts of the reporter insulated from the enhancer, but the remaining bursts still occurred in sync with the other target reporter of the enhancer. These results hint at the importance of higher order chromosome topology in regulating transcriptional activity and highlight the power of multicolor, temporally resolved measurements to understand gene expression regulation.

Another mechanism by which transcription of a gene can be regulated is through the expression of antisense noncoding RNAs. Lenstra and colleagues explored the regulatory function of antisense transcription in yeast by tagging the GAL10 sense transcript with MS2 stem loops and antisense GAL10 ncRNAs with PP7 stem loops (Lenstra et al. 2015). Simultaneous observation of GAL10-MS2 mRNAs and GAL10-PP7 ncRNAs showed that during induction in galactose media, GAL10-MS2 transcription occurred in stochastic bursts that were unaffected by antisense GAL10-PP7 ncRNAs, even when detected simultaneously at the same locus. Inhibition of GAL10-PP7 ncRNA transcription by strand-specific CRSPRi in cells grown in glucose, however, showed that its expression helps to repress leaky transcription of GAL10 and GAL1 in uninduced cells. These experiments showed that expression of an antisense ncRNA can function differently depending on the metabolic state of the cell, indicating that environmental conditions can alter the functionality of ncRNAs.

To directly measure the dynamics of transcription elongation in single living cells, the MS2 and PP7 stem loops can be positioned in the $5^{\prime}$ and $3^{\prime}$ ends of the same gene (Fig. 2B). If the intervening nucleotide sequence between the two labels is sufficiently long, then the time between the appearance of the $5^{\prime}$ and $3^{\prime}$ labels can be used to directly measure the rate of Pol II elongation. In yeast, Hocine and colleagues labeled a reporter driven by the $G A L$ promoter that contained the MDN1 open reading frame $(14.7 \mathrm{~kb})$ flanked by PP7 stem loops in the $5^{\prime}$ end and MS2 stem loops in the $3^{\prime}$ end (Hocine et al. 2013). On induction of GAL transcription with galactose, these investigators measured an average Pol II elongation rate of $25 \pm 2$ bases sec $^{-1}$; 
however, there was considerable cell-to-cell variation with

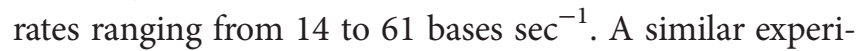
ment in Drosophila embryos gave an average Pol II elonga-

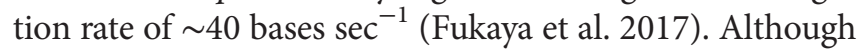
cell-to-cell variation was also observed as in yeast, this rate was not affected by the maternal Bicoid activator gradient as cells across the anterior-posterior axis had similar Pol II elongation rates despite differences in overall transcriptional output. Interestingly, placing the same reporter under the control of the snail enhancer or the inclusion of an intron within the body of a reporter gene resulted in only subtle changes $(<25 \%)$ in Pol II elongation rates. Additionally, in human U2OS osteosarcoma cells, a Ponasterone A inducible promoter was used to induce expression of a reporter containing CFP-24xPP7 stem loops-IRES-Hygromycin24xMS2 stem loops that places the stem loop cassettes $3 \mathrm{~kb}$ apart (Palangat and Larson 2016). In this experiment, the Pol II elongation rate was measured to be faster at 100 bases $\mathrm{sec}^{-1}$. These studies provide a foundation to further explore the regulation of Pol II dynamics, either elongation rate or pausing, by site-specific integration of PP7 and MS2 stem loops into positions within endogenous genes. These single-molecule live cell measurements will enable genespecific cis and trans effects that have been observed in ensemble measurements to be characterized in greater detail (Jonkers and Lis 2015).

\section{SPLICING}

In eukaryotes, intronic sequences must be removed from pre-mRNAs to generate mature transcripts. Using the wellstudied $\beta$-globin gene that contains two introns as a model system, Martin and colleagues inserted either the $\lambda \mathrm{N}$ boxB stem loops or MS2 stem loops into the first or second intron (Martin et al. 2013). This design allowed the splicing of the two introns to be followed independently in real time. These investigators measured different splicing times for the two introns with the first intron taking on average $20 \mathrm{sec}$, whereas the second intron took slightly longer at $30 \mathrm{sec}$. In these experiments, splicing of both introns was observed to finish before release of the transcript from the transcription site. Incorporation of the mouse IgM intron that contains a weak splice site resulted in a longer intron lifetime of $43 \mathrm{sec}$.

A similar approach using the $\beta$-globin gene was also used by Coulon and colleagues, except that PP7 stem loops were placed only into the second intron and MS2 stem loops were inserted into the $3^{\prime}$ UTR of a two-color splicing reporter gene (Fig. 2C) (Coulon et al. 2014). These investigators observed splicing events both before and after release of the pre-mRNA transcript from the transcription site. Their observations are consistent with a kinetic competition between stochastic splicing, elongation, and termination, suggesting that there is no deterministic quality control checkpoint that ensures pre-mRNAs are fully spliced before release. Surprisingly, these investigators found that posttranscriptional splicing of pre-mRNAs in the nucleoplasm is an order of magnitude faster than cotranscriptional splicing of the same intron. Combined, these studies provide a foundation to further explore the regulation of splicing and alternative splicing using multicolored RNA reporters.

Day and colleagues adapted the $\beta$-globin PP7 and MS2 splicing reporter to develop a fluorescence microscopy image-based screen to identify inhibitors of transcription, splicing, and termination (Day et al. 2016). These investigators used a library of small molecules that had been previously identified as inhibitors of chromatin readers and writers to show proof-of-concept for their approach. Several compounds that target bromodomains caused a reduction in the Pol II elongation rate. Although limitations in the sensitivity of high-throughput spinning-disk microscopes may have resulted in many false negatives in these initial experiments, this is not likely to constitute a permanent obstacle to cell-based phenotypic screens using single-molecule RNA imaging.

\section{LOCALIZATION}

The ability to monitor the trafficking and localization of multiple RNA species simultaneously in live cells will enable the mechanisms responsible for establishing and maintaining the polarization of RNAs to be further characterized. To this aim, Abbaszadeh and colleagues have shown the detection of bicoid transcripts labeled with MS2 stem loops and oskar transcripts labeled with PP7 stem loops in Drosophila oocytes (Abbaszadeh and Gavis 2016). In stage 10 oocytes, bicoid-MS2 transcripts are localized to the anterior while oskar- $P P 7$ transcripts are localized to the posterior pole. This approach should be particularly informative for understanding the packaging of multiple mRNA species into granules and to dissect the specific cis-acting elements required for distinct RNA localization patterns.

\section{TRANSLATION}

Ribosomes move along the coding sequence of a transcript to decode the information contained within codons to synthesize proteins. This requires that any RNA-protein complexes (e.g., exon junction complex [EJC]) that are bound to the open reading frame must be displaced by the ribosome during the first round of translation. Consequently, the EJC can be used to specifically identify transcripts that have never been translated (Popp and Maquat 2013). Halstead and colleagues used this principle to design a twocolor fluorescent microscopy methodology that enables the 
translation status of individual transcripts to be quantified in single cells. These investigators referred to this technique as translation RNA imaging by coat protein knockoff (TRICK) (Halstead et al. 2015).

TRICK relies on the detection of fluorescent labels that uniquely mark the coding sequence and the $3^{\prime}$ UTR in different colors. PP7 stem loops were engineered that could be efficiently translated by the ribosome and were placed within coding sequence of an mRNA, whereas MS2 stem loops were positioned in the $3^{\prime}$ UTR (Fig. 3A). Imaging of this reporter transcript in cells that also express the PP7 and MS2 coat proteins fused to spectrally distinct fluorescent proteins (e.g., NLS-PCP-GFP and NLS-MCP-RFP) results in untranslated mRNAs being detected as dual-colored spots (NLS-PCP-GFP and NLS-MCP-RFP). During the first round of translation, the NLS-PCP-GFP signal is re- moved from the coding sequence by the ribosome that is then detected as a single-labeled spot (NLS-MCP-RFP) (Fig, 3B). The incorporation of a nuclear localization sequence into NLS-PCP-GFP maintains a low concentration of unbound fluorescent protein in the cytoplasm, which prevents rebinding of NLS-PCP-GFP after translation. Consequently, TRICK cannot be used to monitor subsequent rounds of translation; however, the recently reported techniques for nascent polypeptide imaging (see Morisaki and Stasevich 2019) enable the dynamics of protein synthesis to be quantified in further detail.

The binary readout of the TRICK assay makes it an extremely sensitive assay for characterizing the spatial and temporal regulation of the initiation of protein synthesis. Halstead and colleagues found no evidence for translation of their TRICK reporter transcripts in the nucleus and that
A
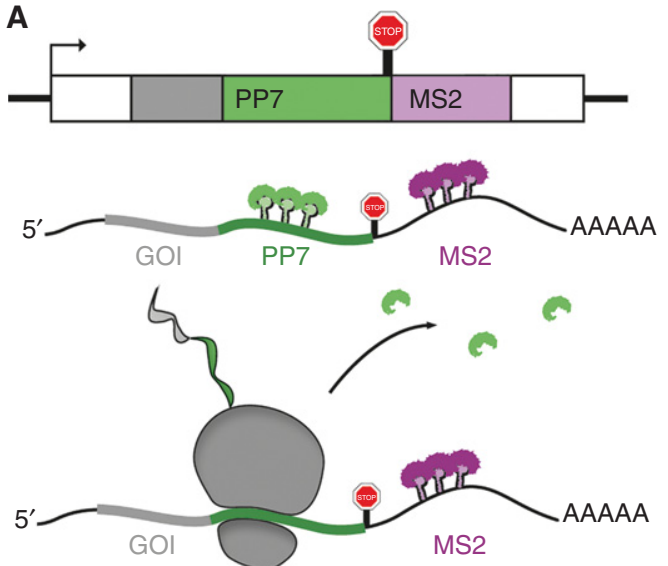

B

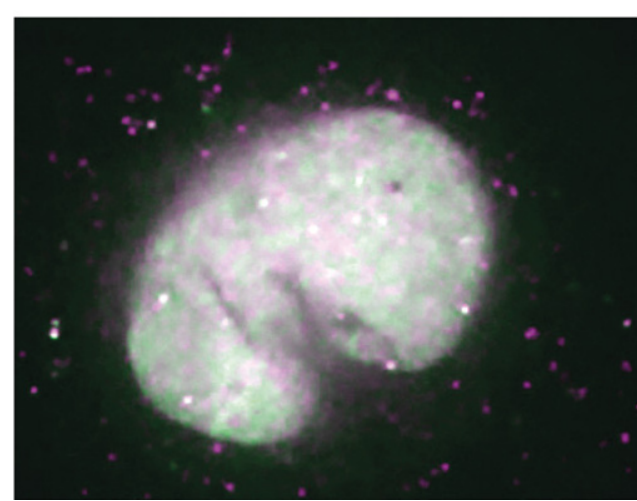

C
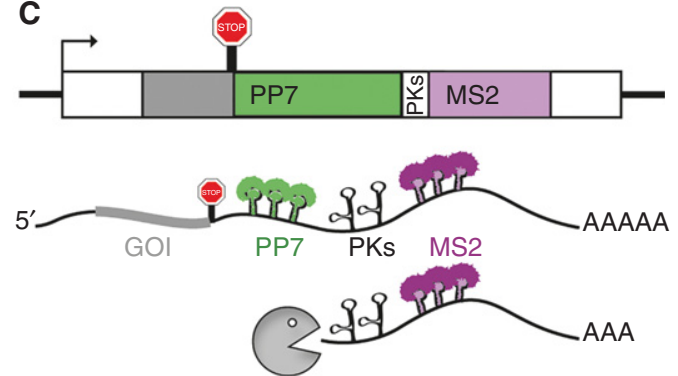

D

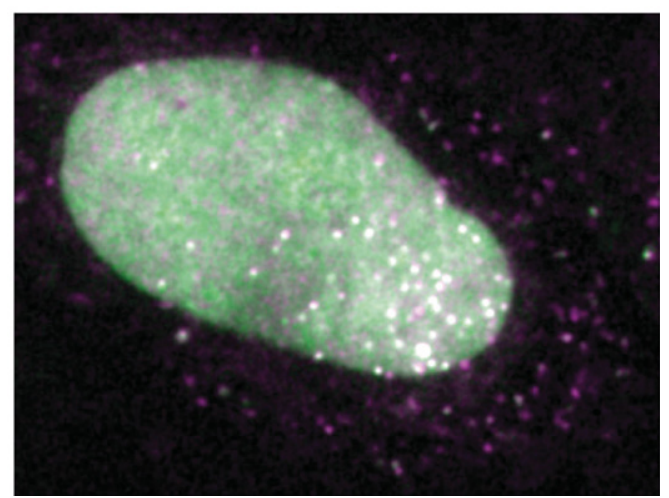

Figure 3. Single-molecule imaging of translation and mRNA decay in the cytoplasm of live cells. $(A)$ Schematic representation of translation RNA imaging by coat protein knockoff (TRICK) for measuring the first round of translation by loss of PP7 label from the coding sequence during the first round of translation. (B) Representative image of TRICK experiment showing untranslated (PP7 and MS2; white) and translated (MS2-only; magenta) messenger RNAs (mRNAs). (C) Schematic representation of 3'-RNA end accumulation during turnover (TREAT) for measuring mRNA degradation using viral pseudoknots to stabilize a decay intermediate. $(D)$ Representative image of TREAT experiment showing intact transcripts (PP7 and MS2; white) and stabilized 3' end decay intermediates (MS2-only; magenta) arising from Xrn1-mediated degradation. 
the first round of translation was distributed through the cytoplasm. The sequestration of mRNAs within messenger ribonucleoprotein (mRNP) granules either during stress maintained the granule-associated transcripts in a translationally repressed state. In Drosophila oocytes, oskar mRNA is localized to the posterior pole at late stages and TRICK confirmed that oskar transcripts are translationally repressed during transport. Voigt and colleagues used TRICK to show that mRNAs encoding secreted proteins are indeed localized to the endoplasmic reticulum (ER) during the first round of translation, which is consistent with the signal recognition particle (SRP)-mediated model for targeting (Voigt et al. 2017). Interestingly, it was also shown that mRNAs encoding cytosolic proteins could similarly be targeted as early as the first round of translation to the ER, albeit at much lower incidence.

\section{3 mRNA DECAY}

The nucleases involved in RNA decay pathways are highly processive enzymes that cause rapid disappearance of degradation intermediates within cells, which has prevented the spatial and temporal dynamics of mRNA turnover from being investigated in detail (Garneau et al. 2007). The direct measurement of mRNA decay by microscopy is additionally challenging because degradation must be unequivocally distinguished from photobleaching or movement of the transcript out of the focal volume that could also result in a loss of signal. Previously, it was shown that small interfering RNA (siRNA)-mediated knockdown of either Xrn1 or the exosome resulted in the accumulation of RISC-generated degradation intermediates that could be detected by northern blot (Orban and Izaurralde 2005). In the case of Xrn1 depletion, the half-life of the $3^{\prime}$-degradation intermediate increased from $>15 \mathrm{~min}$ to $>4 \mathrm{~h}$. This result indicated that these RNA fragments were not immediately recognized as aberrant RNAs within the cell that could be efficiently cleared by alternative decay pathways and suggested that stabilizing degradation intermediates might be an entry point for imaging decay.

Although depletion of the decay machinery precludes studying physiological mRNA decay, a viral RNA pseudoknot structure was identified in flaviviruses that blocks Xrn1 by sequestering the $5^{\prime}$-phosphate from the enzyme, thereby preventing further degradation of the viral genome (Chapman et al. 2014). A single-molecule mRNA turnover biosensor was engineered that contains a $3^{\prime}$ UTR with the tandem pseudoknots from the Kunjin strain of the West Nile virus placed between the orthogonal PP7 and MS2 bacteriophage stem loops (Fig. 3C) (Horvathova et al. 2017). Because the MS2 stem loops are protected from Xrn1-mediated degradation by the pseudoknots, this bio- sensor allows discrimination between intact (PP7 and MS2) transcripts and stabilized $3^{\prime}$ end degradation intermediates (MS2 only) in living cells that express the PCP and MCP fused to spectrally distinct fluorescent proteins (Fig. 3D). Similarly, smFISH probes can be targeted to the two distinct regions of the transcript to image mRNA decay in fixed cells. This technique was named $3^{\prime}$-RNA end accumulation during turnover (TREAT).

Using TREAT, these investigators quantified the dynamics of mRNA degradation in single cells. Although transcription can occur in bursts, mRNA degradation was found to be consistent with a simple Poisson process indicating that individual decay events occur independently in time. Many of the eukaryotic proteins involved in mRNA decay have been found to accumulate in cytosolic structures called P-bodies suggesting that spatial compartmentalization may play a role in degradation (Sheth and Parker 2003). Using TREAT, these investigators did not detect either decay events or the accumulation of degradation intermediates within P-bodies during normal cell growth or stress, indicating that these compartments may not be general sites of mRNA decay.

The addition of a sequence targeted by an siRNA between the PP7 stem loops and the viral pseudoknots enabled monitoring of RISC-mediated slicing of mRNAs. Real-time observation of the severing of a transcript into distinct $5^{\prime}$ and $3^{\prime}$ ends that could be independently tracked revealed that cleavage was not coupled to degradation of the slicing intermediates and that slicing was not detected in the nucleus but throughout the cytosol.

\section{OUTLOOK}

The development of methodologies for imaging single molecules of RNA in fixed and living cells has profoundly altered our understanding of the regulation of this level of gene expression. For instance, biological phenomena such as transcriptional bursting, cellular heterogeneity in transcript abundances, mRNA localization, and localized translation have been uncovered in part because of progress in imaging technologies. These discoveries provide unique access to observe the molecular mechanisms underlying cell fate decisions, morphogenesis, drug resistance, and memory formation. Although the technological accomplishments of the field over the past two decades have been dramatic, there remains considerable room for progress. The throughput of imaging technologies lags far behind the capabilities of sequencing in terms of genomic coverage. Ongoing efforts in multiplexing RNA measurements in fixed cells is increasing at a rapid pace, but applications in live cell imaging have remained limited because of the low number of available orthogonal labeling techniques and spectrally separable fluorophores. Progress in genome editing and alter- 
native approaches for fluorescent labeling of RNAs should drive the field forward. Further developments in technologies able to visualize mRNA dynamics inside living organisms and tissues would certainly prove transformational in our understanding of the processes that regulate gene expression in complex cellular environments.

\section{ACKNOWLEDGMENTS}

This work was supported by the Novartis Research Foundation (J.A.C.), Swiss National Science Foundation (SNF) grant 31003A_156477 (J.A.C.), and the EMBO Young Investigator Program (J.A.C.). We thank F. Voigt for assistance with illustrations.

\section{REFERENCES}

* Reference is also in this collection.

Abbaszadeh EK, Gavis ER. 2016. Fixed and live visualization of RNAs in Drosophila oocytes and embryos. Methods 98: 34-41.

Adivarahan S, Rahman S, Zenklusen D. 2017. Spatial organization of single mRNPs at different stages of the gene expression pathway. bioRxiv doi: 10.1101/237008.

Ashley J, Cordy B, Lucia D, Fradkin LG, Budnik V, Thomson T. 2018. Retrovirus-like gag protein Arc1 binds RNA and traffics across synaptic boutons. Cell 172: 262-274.e11.

Bahar Halpern K, Caspi I, Lemze D, Levy M, Landen S, Elinav E, Ulitsky I, Itzkovitz S. 2015. Nuclear retention of mRNA in mammalian tissues. Cell Rep 13: 2653-2662.

Bai L, Charvin G, Siggia ED, Cross FR. 2010. Nucleosome-depleted regions in cell-cycle-regulated promoters ensure reliable gene expression in every cell cycle. Dev Cell 18: 544-555.

Bartman CR, Hsu SC, Hsiung CC-S, Raj A, Blobel GA. 2016. Enhancer regulation of transcriptional bursting parameters revealed by forced chromatin looping. Mol Cell 62: 237-247.

Batish M, van den Bogaard P, Kramer FR, Tyagi S. 2012. Neuronal mRNAs travel singly into dendrites. Proc Natl Acad Sci 109: 46454650.

Bertrand E, Chartrand P, Schaefer M, Shenoy SM, Singer RH, Long RM. 1998. Localization of ASH1 mRNA particles in living yeast. Mol Cell 2: 437-445.

Buxbaum AR, Wu B, Singer RH. 2014. Single $\beta$-actin mRNA detection in neurons reveals a mechanism for regulating its translatability. Science 343: 419-422.

Cabili MN, Dunagin MC, McClanahan PD, Biaesch A, Padovan-Merhar O, Regev A, Rinn JL, Raj A. 2015. Localization and abundance analysis of human lncRNAs at single-cell and single-molecule resolution. Genome Biol 16: 20.

Chapman EG, Costantino DA, Rabe JL, Moon SL, Wilusz J, Nix JC, Kieft JS. 2014. The structural basis of pathogenic subgenomic flavivirus RNA (sfRNA) production. Science 344: 307-310.

Chen J, Nikolaitchik O, Singh J, Wright A, Bencsics CE, Coffin JM, Ni N, Lockett S, Pathak VK, Hu W-S. 2009. High efficiency of HIV-1 genomic RNA packaging and heterozygote formation revealed by single virion analysis. Proc Natl Acad Sci 106: 13535-13540.

Chen KH, Boettiger AN, Moffitt JR, Wang S, Zhuang X. 2015. RNA imaging. Spatially resolved, highly multiplexed RNA profiling in single cells. Science 348: aaa6090.

Chen F, Wassie AT, Cote AJ, Sinha A, Alon S, Asano S, Daugharthy ER, Chang J-B, Marblestone A, Church GM, et al. 2016. Nanoscale imaging of RNA with expansion microscopy. Nat Methods 13: 679-684.
Cho W-K, Jayanth N, English BP, Inoue T, Owen Andrews J, Conway W, Grimm JB, Spille J-H, Lavis LD, Lionnet T, et al. 2016. RNA polymerase II cluster dynamics predict mRNA output in living cells. eLife Sciences 5: e13617.

Choi HMT, Beck VA, Pierce NA. 2014. Next-generation in situ hybridization chain reaction: Higher gain, lower cost, greater durability. ACS Nano 8: 4284-4294.

Chong S, Chen C, Ge H, Xie XS. 2014. Mechanism of transcriptional bursting in bacteria. Cell 158: 314-326.

Coskun AF, Cai L. 2016. Dense transcript profiling in single cells by image correlation decoding. Nat Methods 13: 657-660.

Coulon A, Ferguson ML, de Turris V, Palangat M, Chow CC, Larson DR. 2014. Kinetic competition during the transcription cycle results in stochastic RNA processing. eLife doi: 10.7554/eLife.03939.

Czaplinski K. 2017. Techniques for single-molecule mRNA imaging in living cells. Adv Exp Med Biol 978: 425-441.

Daigle N, Ellenberg J. 2007. $\lambda_{N^{-}}$GFP: An RNA reporter system for live-cell imaging. Nat Methods 4: 633-636.

Day CR, Chen H, Coulon A, Meier JL, Larson DR. 2016. High-throughput single-molecule screen for small-molecule perturbation of splicing and transcription kinetics. Methods 96: 59-68.

Femino AM, Fay FS, Fogarty K, Singer RH. 1998. Visualization of single RNA transcripts in situ. Science 280: $585-590$.

Fukaya T, Lim B, Levine M. 2016. Enhancer control of transcriptional bursting. Cell 166: 358-368.

Fukaya T, Lim B, Levine M. 2017. Rapid rates of Pol II elongation in the Drosophila embryo. Curr Biol 27: 1387-1391.

Gandhi SJ, Zenklusen D, Lionnet T, Singer RH. 2011. Transcription of functionally related constitutive genes is not coordinated. Nat Struct Mol Biol 18: 27-34.

Garneau NL, Wilusz J, Wilusz CJ. 2007. The highways and byways of mRNA decay. Nat Rev Mol Cell Biol 8: 113-126.

Halstead JM, Lionnet T, Wilbertz JH, Wippich F, Ephrussi A, Singer RH, Chao JA. 2015. Translation. An RNA biosensor for imaging the first round of translation from single cells to living animals. Science 347: 1367-1671.

Harrison PR, Conkie D, Paul J, Jones K. 1973. Localisation of cellular globin messenger RNA by in situ hybridisation to complementary DNA. FEBS Lett 32: 109-112.

Hocine S, Raymond P, Zenklusen D, Chao JA, Singer RH. 2013. Singlemolecule analysis of gene expression using two-color RNA labeling in live yeast. Nat Methods 10: 119-121.

Horvathova I, Voigt F, Kotrys AV, Zhan Y, Artus-Revel CG, Eglinger J, Stadler MB, Giorgetti L, Chao JA. 2017. The dynamics of mRNA turnover revealed by single-molecule imaging in single cells. Mol Cell 68: 615-625.e9.

Jonkers I, Lis JT. 2015. Getting up to speed with transcription elongation by RNA polymerase II. Nat Rev Mol Cell Biol 16: 167-177.

Larson DR, Zenklusen D, Wu B, Chao JA, Singer RH. 2011. Real-time observation of transcription initiation and elongation on an endogenous yeast gene. Science 332: 475-478.

Lécuyer E, Yoshida H, Parthasarathy N, Alm C, Babak T, Cerovina T, Hughes TR, Tomancak P, Krause HM. 2007. Global analysis of mRNA localization reveals a prominent role in organizing cellular architecture and function. Cell 131: 174-187.

Lee JH, Daugharthy ER, Scheiman J, Kalhor R, Yang JL, Ferrante TC, Terry R, Jeanty SS, Li C, Amamoto R, et al. 2014. Highly multiplexed subcellular RNA sequencing in situ. Science 343: 1360-1363.

Lenstra TL, Coulon A, Chow CC, Larson DR. 2015. Single-molecule imaging reveals a switch between spurious and functional ncRNA transcription. Mol Cell 60: 597-610.

Levesque MJ, Raj A. 2013. Single-chromosome transcriptional profiling reveals chromosomal gene expression regulation. Nat Methods 10: 246-248.

Levesque MJ, Ginart P, Wei Y, Raj A. 2013. Visualizing SNVs to quantify allele-specific expression in single cells. Nat Methods 10: 865-867. 
Levsky JM, Shenoy SM, Pezo RC, Singer RH. 2002. Single-cell gene expression profiling. Science 297: 836-840.

Lionnet T, Singer RH. 2012. Transcription goes digital. EMBO Rep 13: 313-321.

Lionnet T, Czaplinski K, Darzacq X, Shav-Tal Y, Wells AL, Chao JA, Park HY, de Turris V, Lopez-Jones M, Singer RH. 2011. A transgenic mouse for in vivo detection of endogenous labeled mRNA. Nat Methods 8: $165-170$

Little SC, Tikhonov M, Gregor T. 2013. Precise developmental gene expression arises from globally stochastic transcriptional activity. Cell 154: 789-800.

Long X, Colonell J, Wong AM, Singer RH, Lionnet T. 2017. Quantitative mRNA imaging throughout the entire Drosophila brain. Nat Methods 14: 703-706.

Lu J, Tsourkas A. 2009. Imaging individual microRNAs in single mammalian cells in situ. Nucleic Acids Res 37: e100.

Lubeck E, Cai L. 2012. Single-cell systems biology by super-resolution imaging and combinatorial labeling. Nat Methods 9: 743-748.

Lubeck E, Coskun AF, Zhiyentayev T, Ahmad M, Cai L. 2014. Single-cell in situ RNA profiling by sequential hybridization. Nat Methods 11: 360-361.

Martin KC, Ephrussi A. 2009. mRNA localization: Gene expression in the spatial dimension. Cell 136: 719-730.

Martin RM, Rino J, Carvalho C, Kirchhausen T, Carmo-Fonseca M. 2013. Live-cell visualization of pre-mRNA splicing with single-molecule sensitivity. Cell Rep 4: 1144-1155.

Mellis IA, Gupte R, Raj A, Rouhanifard SH. 2017. Visualizing adenosineto-inosine RNA editing in single mammalian cells. Nat Methods 14: 801-804.

* Morisaki T, Stasevich TJ. 2019. Quantifying single mRNA translation kinetics in living cells. Cold Spring Harb Persp Biol 11: a032078.

Mueller F, Senecal A, Tantale K, Marie-Nelly H, Ly N, Collin O, Basyuk E, Bertrand E, Darzacq X, Zimmer C. 2013. FISH-quant: Automatic counting of transcripts in 3D FISH images. Nat Methods 10: 277-278.

Nishikura K. 2010. Functions and regulation of RNA editing by ADAR deaminases. Annu Rev Biochem 79: 321-349.

Ochiai H, Sugawara T, Sakuma T, Yamamoto T. 2014. Stochastic promoter activation affects Nanog expression variability in mouse embryonic stem cells. Sci Rep 4: 7125.

Orban TI, Izaurralde E. 2005. Decay of mRNAs targeted by RISC requires XRN1, the Ski complex, and the exosome. RNA 11: 459-469.

Palangat M, Larson DR. 2016. Single-gene dual-color reporter cell line to analyze RNA synthesis in vivo. Methods 103: 77-85.

Pastuzyn ED, Day CE, Kearns RB, Kyrke-Smith M, Taibi AV, McCormick J, Yoder N, Belnap DM, Erlendsson S, Morado DR, et al. 2018. The neuronal gene Arc encodes a repurposed retrotransposon Gag protein that mediates intercellular RNA transfer. Cell 172: 275-288.e18.

Popp MW-L, Maquat LE. 2013. Organizing principles of mammalian nonsense-mediated mRNA decay. Annu Rev Genet 47: 139-165.

Raj A, Peskin CS, Tranchina D, Vargas DY, Tyagi S. 2006. Stochastic mRNA synthesis in mammalian cells. PLoS Biol 4: e309.

Raj A, van den Bogaard P, Rifkin SA, van Oudenaarden A, Tyagi S. 2008. Imaging individual mRNA molecules using multiple singly labeled probes. Nat Methods 5: 877-879.

Renwick N, Cekan P, Masry PA, McGeary SE, Miller JB, Hafner M, Li Z, Mihailovic A, Morozov P, Brown M, et al. 2013. Multicolor microRNA FISH effectively differentiates tumor types. J Clin Invest 123: 26942702.

Richardson DS, Lichtman JW. 2015. Clarifying tissue clearing. Cell 162: 246-257.
Satija R, Farrell JA, Gennert D, Schier AF, Regev A. 2015. Spatial reconstruction of single-cell gene expression data. Nat Biotechnol 33: 495502.

Shaffer SM, Dunagin MC, Torborg SR, Torre EA, Emert B, Krepler C, Beqiri M, Sproesser K, Brafford PA, Xiao M, et al. 2017. Rare cell variability and drug-induced reprogramming as a mode of cancer drug resistance. Nature 546: 431-435.

Shah S, Lubeck E, Schwarzkopf M, He T-F, Greenbaum A, Sohn CH, Lignell A, Choi HMT, Gradinaru V, Pierce NA, et al. 2016a. Singlemolecule RNA detection at depth by hybridization chain reaction and tissue hydrogel embedding and clearing. Development 143: 28622867.

Shah S, Lubeck E, Zhou W, Cai L. 2016b. In situ transcription profiling of single cells reveals spatial organization of cells in the mouse hippocampus. Neuron 92: 342-357.

Sheth U, Parker R. 2003. Decapping and decay of messenger RNA occur in cytoplasmic processing bodies. Science 300: 805-808.

Shin Y, Brangwynne CP. 2017. Liquid phase condensation in cell physiology and disease. Science 357: aaf4382.

Ståhl PL, Salmén F, Vickovic S, Lundmark A, Navarro JF, Magnusson J, Giacomello S, Asp M, Westholm JO, Huss M, et al. 2016. Visualization and analysis of gene expression in tissue sections by spatial transcriptomics. Science 353: 78-82.

Stoeger T, Battich N, Pelkmans L. 2016. Passive noise filtering by cellular compartmentalization. Cell 164: 1151-1161.

Sydor AM, Czymmek KJ, Puchner EM, Mennella V. 2015. Super-resolution microscopy: From single molecules to supramolecular assemblies. Trends Cell Biol 25: 730-748.

Takizawa PA, Vale RD. 2000. The myosin motor, Myo4p, binds Ash1 mRNA via the adapter protein, She3p. Proc Natl Acad Sci 97: 52735278.

Taniguchi Y, Choi PJ, Li GW, Chen H, Babu M, Hearn J, Emili A, Xie XS. 2010. Quantifying E. coli proteome and transcriptome with singlemolecule sensitivity in single cells. Science 329: 533-538.

Trcek T, Grosch M, York A, Shroff H, Lionnet T, Lehmann R. 2015. Drosophila germ granules are structured and contain homotypic mRNA clusters. Nat Commun 6: 7962.

Trcek T, Lionnet T, Shroff H, Lehmann R. 2017. mRNA quantification using single-molecule FISH in Drosophila embryos. Nat Protoc 12: 1326-1348.

Tutucci E, Livingston NM, Singer RH, Wu B. 2018. Imaging mRNA in vivo, from birth to death. Annu Rev Biophys doi: 10.1146/annurevbiophys-070317-033037.

Vargas DY, Shah K, Batish M, Levandoski M, Sinha S, Marras SA, Schedl P, Tyagi S. 2011. Single-molecule imaging of transcriptionally coupled and uncoupled splicing. Cell 147: 1054-1065.

Voigt F, Zhang H, Cui XA, Triebold D, Liu AX, Eglinger J, Lee ES, Chao JA, Palazzo AF. 2017. Single-molecule quantification of translationdependent association of mRNAs with the endoplasmic reticulum. Cell Rep 21: 3740-3753.

Waks Z, Klein AM, Silver PA. 2011. Cell-to-cell variability of alternative RNA splicing. Mol Syst Biol 7: 506.

Wang F, Flanagan J, Su N, Wang L-C, Bui S, Nielson A, Wu X, Vo H-T, Ma X-J, Luo Y. 2012. RNAscope: A novel in situ RNA analysis platform for formalin-fixed, paraffin-embedded tissues. J Mol Diagn 14: $22-29$.

Wells SE, Hillner PE, Vale RD, Sachs AB. 1998. Circularization of mRNA by eukaryotic translation initiation factors. Mol Cell 2: 135-140.

Zenklusen D, Larson DR, Singer RH. 2008. Single-RNA counting reveals alternative modes of gene expression in yeast. Nat Struct Mol Biol 15: $1263-1271$. 


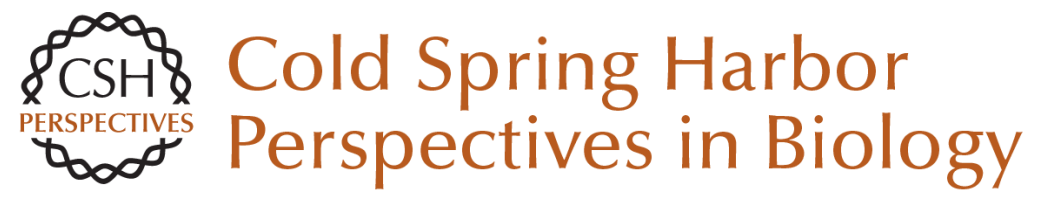

\section{Imaging the Life and Death of mRNAs in Single Cells}

Jeffrey A. Chao and Timothée Lionnet

Cold Spring Harb Perspect Biol 2018; doi: 10.1101/cshperspect.a032086

Subject Collection RNA Worlds

Alternate RNA Structures

Marie Teng-Pei Wu and Victoria D'Souza

Approaches for Understanding the Mechanisms

of Long Noncoding RNA Regulation of Gene

Expression

Patrick McDonel and Mitchell Guttman

Principles and Practices of Hybridization Capture

Experiments to Study Long Noncoding RNAs That

Act on Chromatin

Matthew D. Simon and Martin Machyna

Linking RNA Sequence, Structure, and Function

on Massively Parallel High-Throughput

Sequencers

Sarah K. Denny and William J. Greenleaf

Extensions, Extra Factors, and Extreme

Complexity: Ribosomal Structures Provide

Insights into Eukaryotic Translation

Melanie Weisser and Nenad Ban

Nascent RNA and the Coordination of Splicing with Transcription

Karla M. Neugebauer

Combining Mass Spectrometry (MS) and Nuclear Magnetic Resonance (NMR) Spectroscopy for Integrative Structural Biology of Protein-RNA Complexes

Alexander Leitner, Georg Dorn and Frédéric H.-T. Allain
Structural Biology of Telomerase

Yaqiang Wang, Lukas Susac and Juli Feigon

Structural Insights into Nuclear pre-mRNA

Splicing in Higher Eukaryotes

Berthold Kastner, Cindy L. Will, Holger Stark, et al.

What Are 3' UTRs Doing?

Christine Mayr

Single-Molecule Analysis of Reverse

Transcriptase Enzymes

Linnea I. Jansson and Michael D. Stone

CRISPR Tools for Systematic Studies of RNA

Regulation

Jesse Engreitz, Omar Abudayyeh, Jonathan

Gootenberg, et al.

Relating Structure and Dynamics in RNA Biology Kevin P. Larsen, Junhong Choi, Arjun Prabhakar, et al.

Beyond DNA and RNA: The Expanding Toolbox of Synthetic Genetics

Alexander I. Taylor, Gillian Houlihan and Philipp Holliger

For additional articles in this collection, see http://cshperspectives.cshlp.org/cgi/collection/

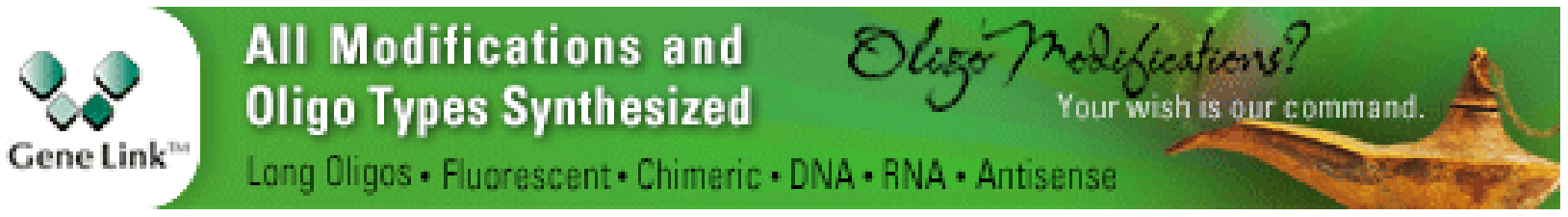

Copyright @ 2018 Cold Spring Harbor Laboratory Press; all rights reserved 
Discovering and Mapping the Modified Nucleotides That Comprise the Epitranscriptome of mRNA

Bastian Linder and Samie R. Jaffrey
Structural Basis of Nuclear pre-mRNA Splicing:

\section{Lessons from Yeast}

Clemens Plaschka, Andrew J. Newman and Kiyoshi Nagai

For additional articles in this collection, see http://cshperspectives.cshlp.org/cgi/collection/

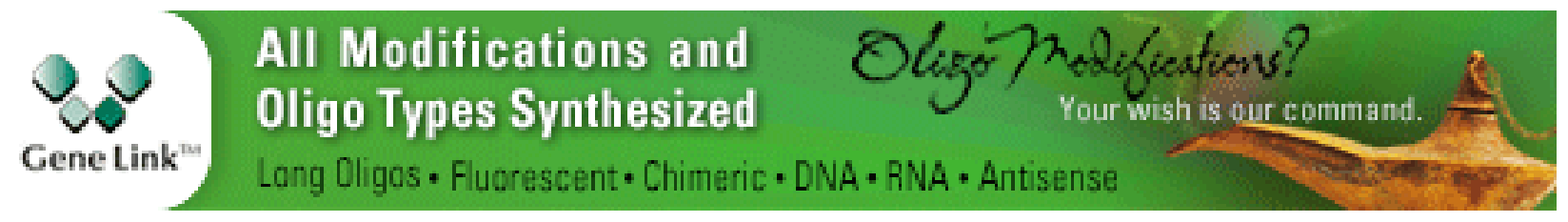

Copyright (C) 2018 Cold Spring Harbor Laboratory Press; all rights reserved 